UCRL-ID-125796

\title{
LCR Bridge Measurements at Elevated Operating Levels
}

\author{
Michael J. Wilson \\ David A. Goerz \\ Ron D. Speer
}

December 1997

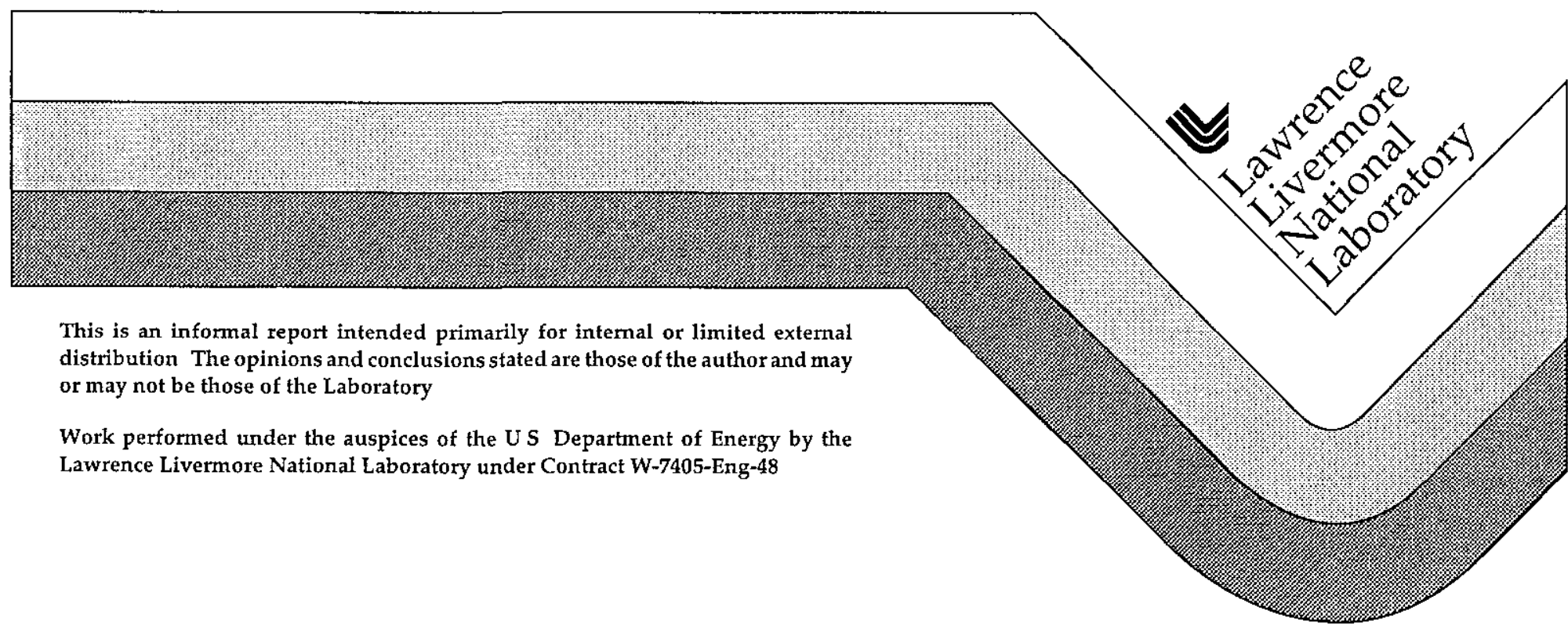




\section{DISCLAIMER}

This document was prepared as an account of work sponsored by an agency of the United States Government. Neither the United States Government nor the University of California nor any of their employees, makes any warranty, express or implied, or assumes any legal liability or responsibility for the accuracy, completeness, or usefulness of any information, apparatus, product, or process disclosed, or represents that its use would not infringe privately owned rights. Reference herein to any specific commercial product, process, or service by trade name, trademark, manufacturer, or otherwise, does not necessarily constitute or imply its endorsement, recommendation, or favoring by the United States Government or the University of California. The views and opinions of authors expressed herein do not necessarily state or reflect those of the United States Government or the University of California, and shall not be used for advertising or product endorsement purposes.

This report has been reproduced directly from the best available copy.

Available to DOE and DOE contractors from the Office of Scientific and Technical Information

P.O. Box 62, Oak Ridge, TN 37831

Prices available from (615) 576-8401, FTS 626-8401

Available to the public from the

National Technical Information Service

U.S. Department of Commerce

5285 Port Royal Rd.,

Springfield, VA 22161 


\title{
LCR Bridge Measurements at Elevated Operating Levels
}

\author{
Michael J. Wilson \\ David A. Goexz \\ Ron D. Speer
}

December 1997

, 


\section{EXECUTIVE SUMMARY}

A critical part in the design of high voltage systems is the selection of the appropriate discrete components The proper selection involves consideration to the basic equivalent electrical parameters of inductance, capacitance, and resistance. The typical approach to establish these parameters involves the use of low level measurements at reduce scale and extrapolate the results to the desired operating ranges. A test voltage level of one volt represents the typical low-level measurement system. The linearity of this process is always in question and may not represent the system operating parameters. The following technique involves the use of a standard Hewlett Packard LCR bridge to measure the electrical parameters of a selected component while it is at its operating level. This process not only provides discrete component values at operating stress but also provides limited information of frequency information at the internal frequency steps. Capacitance is the principal parameter of interest and is highly stress related The significance of a varying capacitance as a function of voltage is the direct relationship to the available stored energy Additional concerns of system frequency response depend on the application 


\section{Table of Contents}

Section

Title

Page

Executive summary . .... . ........................................ . . . . .......... . 2

Table of contents ...... . . . . . . . . ............................ . 3

Abstract............................................... . ..................... . . . 4

1.0 Introduction. .. . ....... . ...... . .................................... 4

2.0 Device $u n d e r$ test ........................................ . . .................... . 5

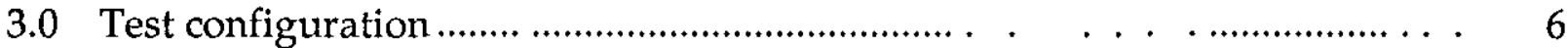

4.0 Representative data of selected components ........................ .......... 7

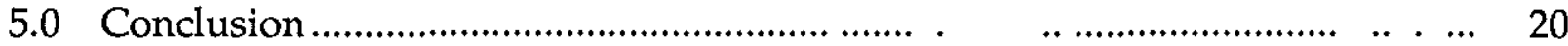

6.0 References............................................................................. .... 20 


\section{Abstract}

This report ${ }^{1}$ describes a method that can be used to measure the dielectric constant of full-scale capacitors at elevated operating levels Capacitors are a critical component in nearly all high-voltage systems. Most often, capacitance values are specified for a static state; but experienced designers know that capacitance can change dramatically at elevated voltage levels ${ }^{2}$ This is particularly evident with most the high-permittivity, ferroelectric ceramics, and it is especially important when relying on these devices for energy storage. Typically, manufactures provide information about their devices by describing the percent change of capacitance at their rated voltage. In some applications devices are operated beyond their rated voltage in order to achieve a higher performance system. This report describes the apparatus and steps used to characterize commercially available ceramic capacitors at voltage levels up to $90 \mathrm{kV}$. Data is presented on various types of ceramic materials including Z5, N4700, SR4700, N2200, and X7R. For better comparison the data is shown as a function of electric field stress in the material.

\section{Introduction}

Capacitors are important as energy storage devices in most pulsed power systems. Capacitors are simply defined by the direct relationship of the permittivity $(\varepsilon)$ of the media separating two electrodes times the ratio of the surface area $(A)$ to the separation distance $(d)$ of the electrodes. The following equation simplifies the relationship.

$$
\begin{gathered}
C=\frac{\varepsilon A}{d}[\mathrm{~F}] \\
\varepsilon=\varepsilon_{\mathrm{R}} \varepsilon_{\mathrm{O}}[\mathrm{F} / \mathrm{cm}]
\end{gathered}
$$

The permittivity of free space is represented by $\varepsilon_{0}$. The relative dielectric constant of the separating media is represented by $\varepsilon_{\mathrm{R}}$ or $k_{e^{\prime}}$ and is typically referred to as the dielectric constant of the material.

where the mks units are:

$$
\begin{aligned}
& \varepsilon_{\mathrm{O}}=885 \times 10^{-14}[\mathrm{~F} / \mathrm{cm}] \\
& A \text { in } \mathrm{cm}^{2} \\
& d \text { in cm }
\end{aligned}
$$

The obvious material to construct a capacitor out of would have a high permittivity. Ideal electrical components have a consistent response over the entire range of all operating environments. High $\varepsilon$ materials having values three orders of magnitude above $\varepsilon_{\mathrm{o}}$ provide attractive dielectric candidates for capacitors. Materials such as ceramics have high $\varepsilon$ and fall into a category of ferroelectric material and as such provide additional design considerations. When the voltage across a typical 
ferroelectric type ceramic device increases the dielectric constant $k_{e}$ decreases The obvious result is a decrease in the capacitance and a reflection on the design circuit parameters. Critical systems requiring specific values of capacitance require that the resulting dielectric constant be known for the operating range.

Manufactures are aware of this effect and provide helpful information on the relative capacitance as a function of operating voltage. Remaining within the guidelines of the manufacture specifications is a conservative design approach and provides some indication of the characteristics of the selected material under elevated stress levels. By repackaging ${ }^{3,4}$ bulk ceramic material operating levels exceeding those referenced by the manufacture are possible. The results of elevated stress on these optimized structures provide for high performance circuits finely tuned for specific parameters.

The classical approach to establish the operating characteristics of variable electrical parameters requires the use of complex custom test circuits These circuits include a variety of charging and discharging scenarios, requiring extensive voltage and current monitors to measure the resulting circuit parameters. Reduced specimen sizes to achieve average field stress similar to the final part have also been employed with success. Modest bias voltages are available as options to high end LCR bridge measuring devices These levels are typically up to 300 volts. Consider the average stress across a reasonable test specimen of $1 \mathrm{~mm}$ and the observed fields are only 3 $\mathrm{kV} / \mathrm{cm}$. This represents a factor of 40 below reasonable material intrinsic breakdown levels of $120 \mathrm{kV} / \mathrm{cm}$

To reduce the time to market for hybrid, high-performance, highly stressed dielectric material an alternative means of measuring the elevated electrical parameters has been demonstrated This process involves the use of a standard low voltage LCR bridge measuring equipment to measure selected materials at the design voltage level. In addition the means of expressing the functional relationship of dielectric constant as a function of average field stress is proposed. The advantage of this relationship provides added value to the measurement and allows a general application of the material characteristics to other applications

\subsection{Device under test}

The initial interest in accessing the functional response of dielectric constant verse field stress was made on standard ceramic products. The two families of materials used for this evaluation were Barium Titanate (Z5V) and Strontium Titanate (N4700). Barium Titanate has a generally accepted loss in energy storage as a function of voltage and available literature indicated only a slight loss with Strontium Titanate As mention above using the material above the reported literature did not provide the required information on the rate of decreased. In addition to these issue custom capacitor houses were solicited to provide specialized packaging. The initial volume of anticipated components did not provide enough interest to the large manufacturing houses. Each 
of the manufactures claimed to be able to provide similar values and quality as compared to other vendors As a result of the data shown in section 40 general trends are observed but differences in specimens are typical. The schematic representation for the device under test (DUT) is shown in Figure 30 as C2.

\subsection{Test configuration}

A test configuration has been employed to elevate the DUT to design levels of stress The configuration provides a versatile holding fixture, a DC blocking capacitor, protection devices, a high-voltage power supply, a low level LCR Bridge, and a fiber optic interconnection to a computer based data acquisition system. In addition the entire fixture is located in a pressurized test fixture enclosure capable of 7-atmosphere pressure. The dimensional range of specimens is $16 \mathrm{~cm}$ thick having a radius of $5 \mathrm{~cm}$. The selected DC blocking capacitor is provided by two series Maxwell $40 \mathrm{nF}, 100 \mathrm{kV}$, series $S, 20 \mathrm{nH}$ capacitors. The principal protection device is a gas filled spark gaps model 3P-4L1 with $51 \Omega$ series voltage-dropping resistors. Additional protection of the bridge was not possible due to the resulting excessive shunting capacitance that would have been required by the desired transorb device. The high-voltage power supply used to bias the DUT to desired stress levels is a remotely controlled Glassman $125 \mathrm{kV}$, $5 \mathrm{~mA}$, series WK. The test fixture includes 2 each $100 \mathrm{k} \Omega$ resistors in series with the blocking capacitor (C1) and the DUT (C2) These two resistors provide the current return path for the bias supply The LCR bridge selected for this measurement is a remotely controlled Hewlett Packard model HP-4275A using the low inductance model HP-16047A test fixture The control and data acquisition is provided by a fiber optically interfaced control link to an IBM computer control system. The applied voltage is remotely adjusted and held at incremental stress levels, while a Lab-View software based routine measures all the available electrical parameter from the DUT. This information is stored in an Excel formatted spreadsheet for analysis. A block diagram of the circuit is provided in Figure 30 . Figure 31 and Figure 32 illustrate the overall and internal views of the test configuration

Figure 3.0 Shown in the adjacent figure is a block diagram representing the circuit and interconnection used to measure the parameters of selected capacitor material at elevated stress levels.

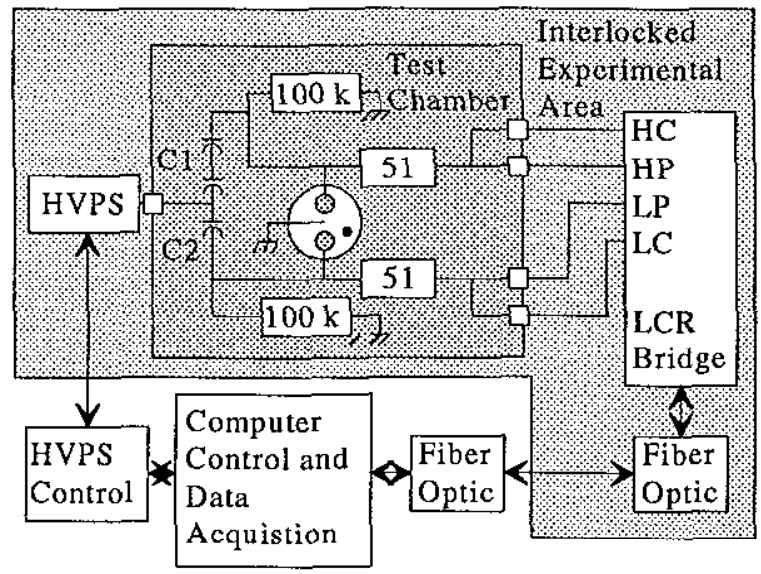




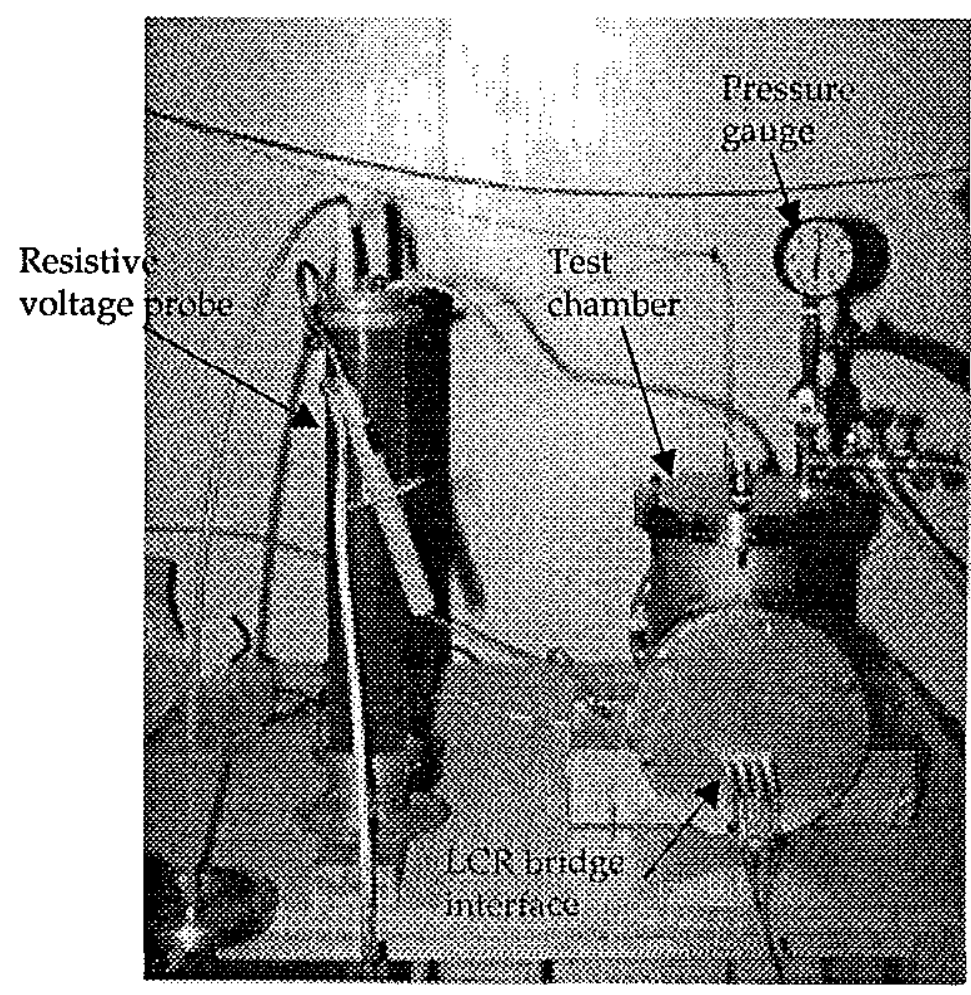

Figure 3.1 Illustrated in the adjacent photo is an overview of the test chamber. Shown are the resistive highvoltage probe and feed through, the test chamber, a bipolar atmospheric pressure gauge, and the coaxial termination for measuring the desired electrical parameters. A reduction in the amount of insulating gas needed for testing is achieved by evacuating the chamber before filling it to 5 atmosphere of SF6

Figure 3.2 An internal view of the test chamber shows the blocking capacitor $\mathrm{C} 1$ and the fixture to mount the component $(\mathrm{C} 2)$ to be evaluated. Not shown are the protection devices used to protect the LCR Bridge These units are located on the attachment side of the feed-through interface connectors to provide a fast response in fault scenarios.

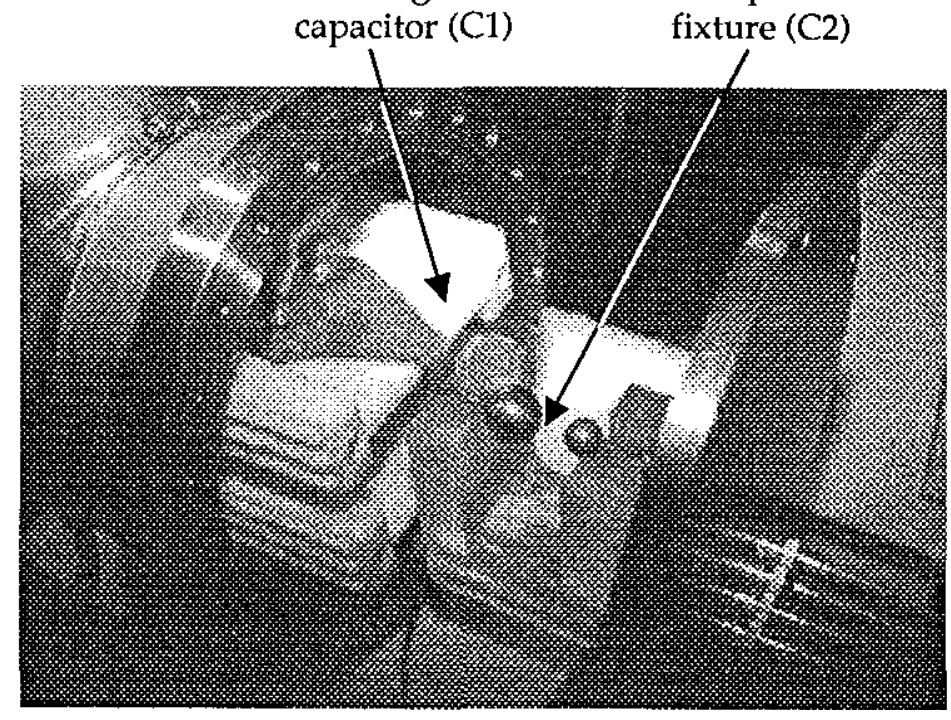

\subsection{Representative data of selected components}

During the process of data analysis it is always necessary to determine the level of confidence in the measurement being taken The question of how well does the measured parameter reflect the desired dimension of interest In this case most of the reported information given at the front panel display is related to a minimal of electrically measure effects. All the front panel (FP) indications are available in digital format through the GPIB buss but only a few of these parameters are necessary to 
reestablish the FP indications. Four parameters are required to provide the desired function. A bridge will measure voltage, current, phase angle, and frequency These are the dimensions that are used to provide the FP displayed information. From these measurements real and imaginary vectors can be derived to establish the basic electrical parameters of the DUT. The critical issue in making elevated measurement is the ability to null out or account for the unwanted yet measured parameters that interfere with the desired parameter. The bridge itself is not capable of compensating for the required test configuration. The test configuration must therefore be dealt with externally and subtracted from the final measurements. To accomplish this the Maxwell capacitors are removed from the circuit and replaced with surrogate termination headers that provide representative inductance. All parameters are measured and used to establish the test fixture baseline. Figure 40 illustrates the relationship of the measured values used to null out the test fixture and measures the desired component

Figure 40 The adjacent figure provides a visual vector view of the various components and shows their relationship to each other in a two dimensional realimaginary plane. The convention of $\pm i$ being imaginary is replaced with $\pm j$ to remove confusion in its use for current

A real-imaginary relationship best describes the impedance $(Z)(\Omega)$ contribution of the three basic electrical equivalent components, resistance $(\mathrm{R})$,

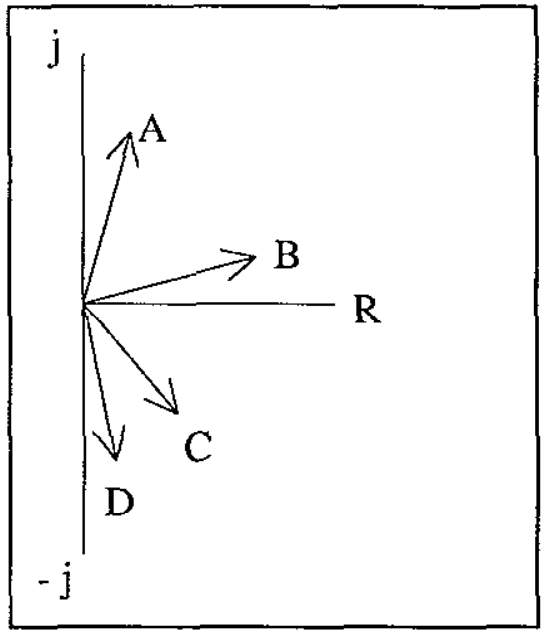
inductive reactance $(j)(\omega \mathrm{L})$, and capacitive reactance $(-j)(1 / \omega C)$ The resulting $Z$ vector rotation $(A, B, C, D)$ from the origin will range within \pm 90 degrees and is referred to as the phase angle $(\phi)$. A purely inductive component having 0 resistance will have a $\phi$ of $90^{\circ}$ and an ideal capacitor will have a $\phi$ of $-90^{\circ}$ The equivalent (effective) series resistance (ESR) reflects the loss vector, $R$, which results in $\phi$ values less than $90^{\circ}$ for the impedance component. The dissipation factor (DF) is the ratio of $\mathrm{R}$ to the reactive component $\omega \mathrm{L}$ or $(\omega \mathrm{C})^{-1}$.

Voltage $(\mathrm{V})$, current $(\mathrm{I})$, phase angle $(\phi)$, and frequency $(\mathrm{Hz})$ are the four critical parameters needed to establish the dimensions of any given electrical component. FP indications reflecting the $\mathrm{V}$ and $\mathrm{I}$ of the instrument are not usually provided. Using the remote GPIB data bus the initial process verified the reported $Z$ to reflect the proper ratio of $\mathrm{V} / \mathrm{I}$. The acceptance of $\mathrm{Z}, \phi$, and $\mathrm{Hz}$ being the indication of the evaluated component allows principal reactive and resistive values to be determined. By measuring each of these components in the test fixture it is possible to establish an origin from which an unknown component value can be characterized This process extends the capability of the unit but is still limited to the internal impedance of the 
selected LCR Bridge. As the test fixture with DUT approaches the impedance of the bridge the nominal source voltage of $1 \mathrm{~V}$ is insufficient to drive enough current into the total fixture-DUT Lower impedance measuring instruments would extend the frequency range but not necessarily the value of the measurement.

Verification of the ability to cancel the baseline from the final measurement is shown by comparing a typical measured capacitor to the same unit in the elevated test fixture. A reasonable accuracy of $5 \%$ is more than adequate for most of these applications. Consider that each electrical component (LCR) requires four different parameters and has only one common component. An error estimate using a 1\% discrete measurement scenario provides a square root of the sum of the squares relationship error estimate of $3.2 \%$. Demonstration of the ability to successfully subtract the test fixture from the measurement is shown by using a sample capacitor and measuring it in a low inductance fixture to establish its value Installing it into the elevated test fixture measuring it again and comparing the results The relative error had a maximum value of $3.03 \%$ over a test frequency of $10 \mathrm{kHz}$ to $400 \mathrm{kHz}$ This is well within our tolerance expectations The source impedance effects are observed at the $1 \mathrm{MHz}$ level when an error of $33 \%$ is observed

\section{Test Results}

The critical issue of concern addressed by this effort is the effective stored energy of selected capacitive material at elevated stress levels Additional information of ESR on selected material has not been included.

The following illustrates several types of investigated ceramic material The first set of data includes a second order correction for a curve fit to the data. All the data includes a higher order curve fit to provide and indication of the functional relationship over the available data. The specific manufactures of the supplied material specimens have been generalized and are indicated by an alphanumeric designator. A summary of all the reported data clearly indicates that even similar materials have different functional relationships. 
4.1 Manufacture A material X7R corrected data

Figure 4.1.1 The following figure illustrates the curve associated with manufacture $\mathbf{A}$ ceramic material type X7R having a typical $k_{e}$ of 1850 . The functional relationship is $k_{e}$ as a function of average field stress (E) in $\mathrm{kV} / \mathrm{cm}$ of a selected specimen. The DUT failed at an average $\mathrm{E}$ of $138 \mathrm{kV} / \mathrm{cm}$. The vertical displacement is in dielectric constant and the horizontal displacement $\mathrm{E}$ is in $\mathrm{kV} / \mathrm{cm}$.

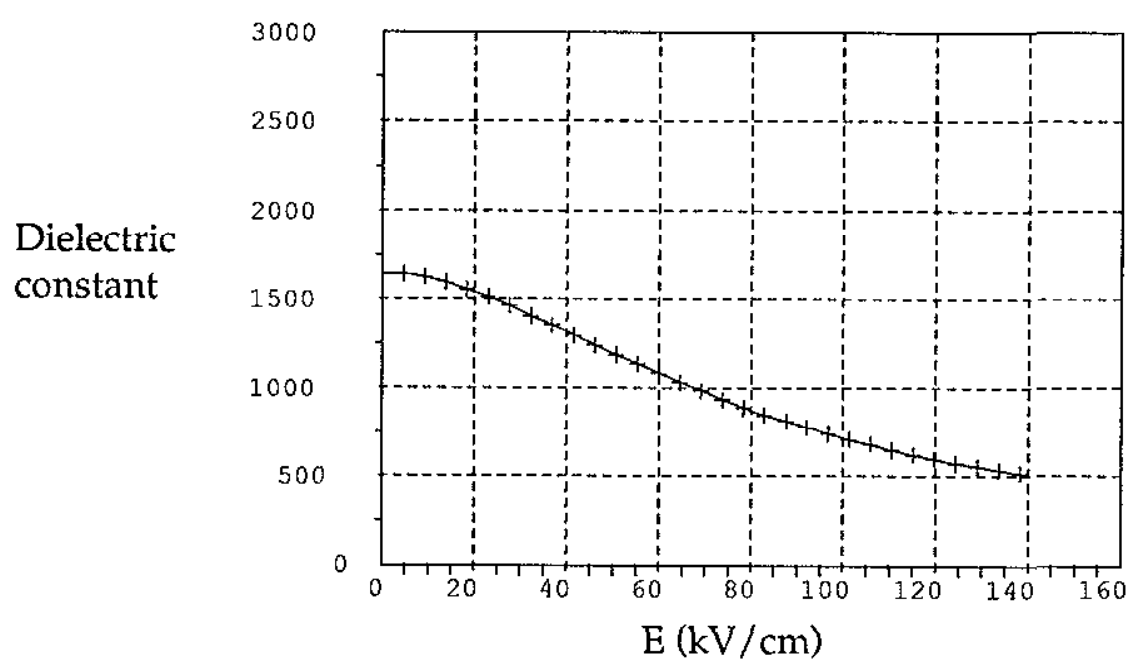

Figure 4.1.2 The following provides figure illustrates a second order curve fit to the above data As a result the algorithm is only reasonable above $10 \mathrm{kV} / \mathrm{cm}$ stress levels

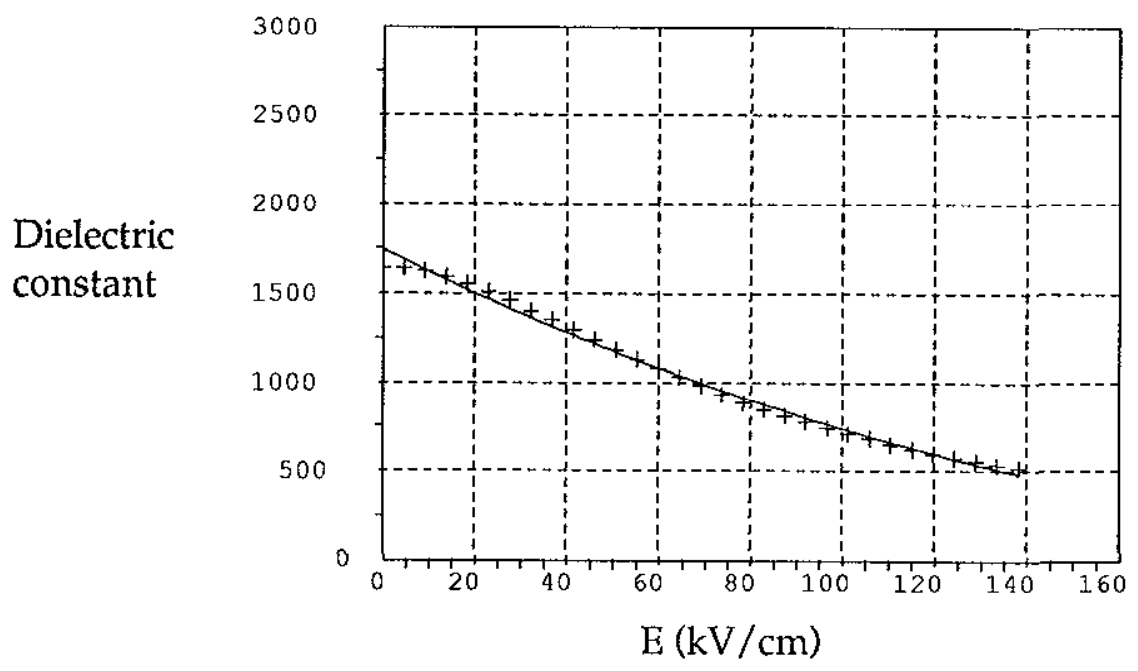

Where the curve is defined as:

$$
\begin{aligned}
& y=A+B x+C x^{2} \\
& A=174177 X 10^{3} \\
& B=-12464 X 10^{1} \\
& C=2.4 X 10^{-2}
\end{aligned}
$$


Figure 4.1.3 The following figure provides a fifth order curve fit to the above data and as a result more closely represents the anticipated values over the measured range.

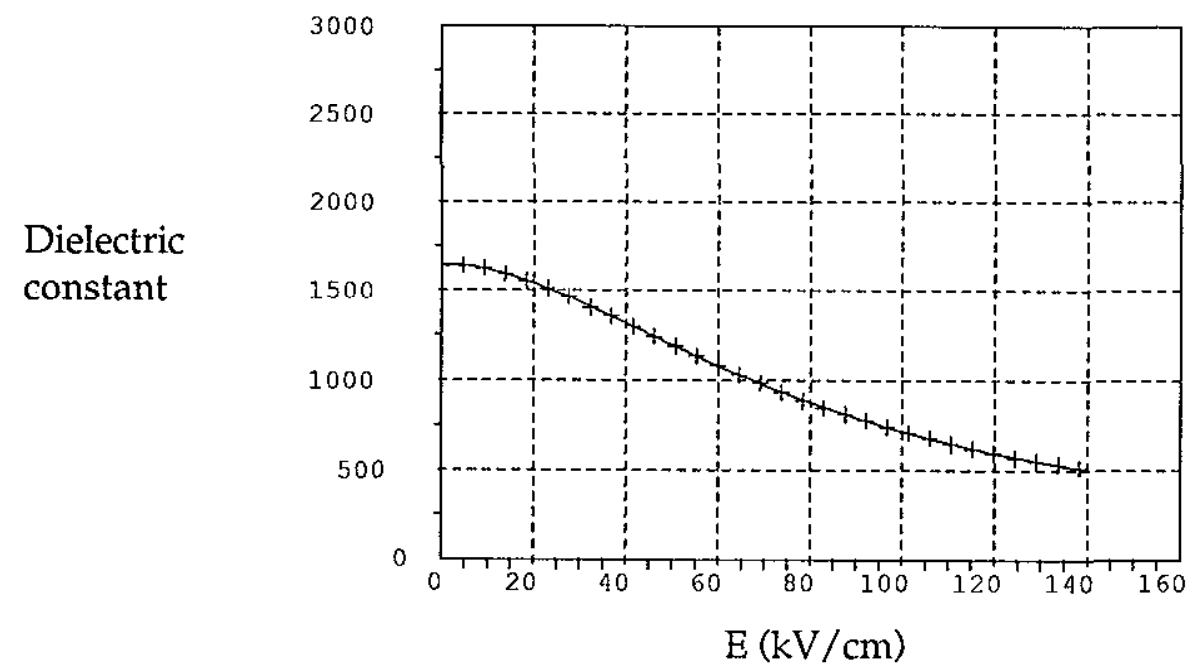

Where the curve is defined as

$$
\begin{aligned}
& y=A+B x+C x^{2}+D x^{3}+E x^{4}+F x^{5} \\
& A=164357 X 10^{3} \\
& B=8.11609 \times 10^{-1} \\
& C=-3.89274 X 10^{-1} \\
& D=5.14737 X 10^{-3} \\
& E=-288281 X 10^{-5} \\
& F=6.19883 \times 10^{-8}
\end{aligned}
$$




\subsection{Manufacture A material Z5 corrected data}

Figure 4.2.1 The following figure illustrates the curve associated with manufacture $\mathbf{A}$ ceramic material type Z5 having a typical $k_{\varepsilon}$ of 3000 The functional relationship is $k_{e}$ as a function of average field stress (E) in $\mathrm{kV} / \mathrm{cm}$ of a selected specimen The DUT failed at an average $E$ of $80 \mathrm{kV} / \mathrm{cm}$. The vertical displacement is in dielectric constant and the horizontal displacement $\mathrm{E}$ is in $\mathrm{kV} / \mathrm{cm}$.

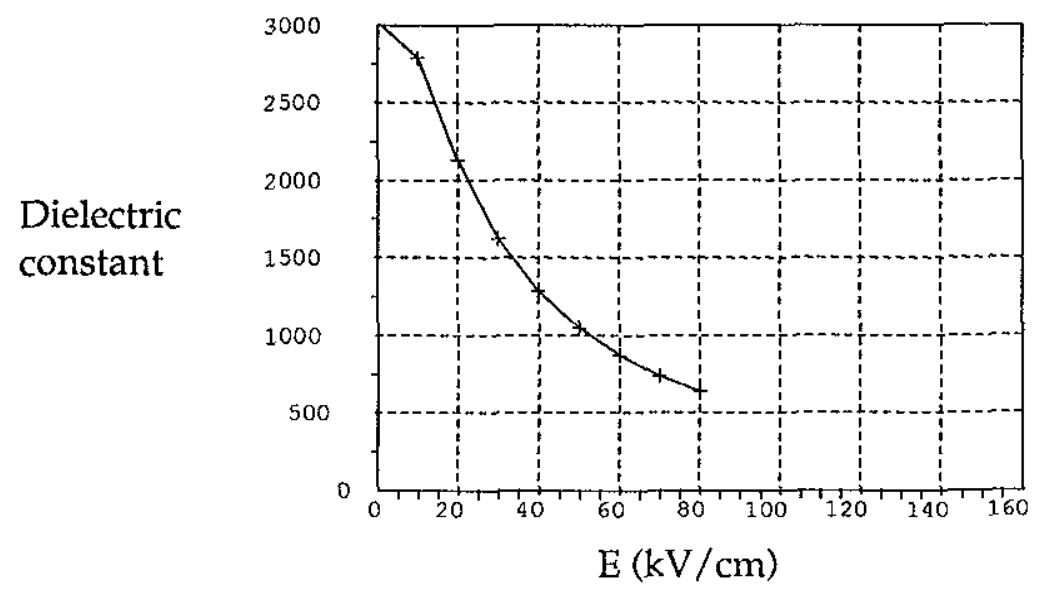

Figure 4.22 The following figure provides a fifth order curve fit to the above data and as a result more closely represents the anticipated values over the measured range

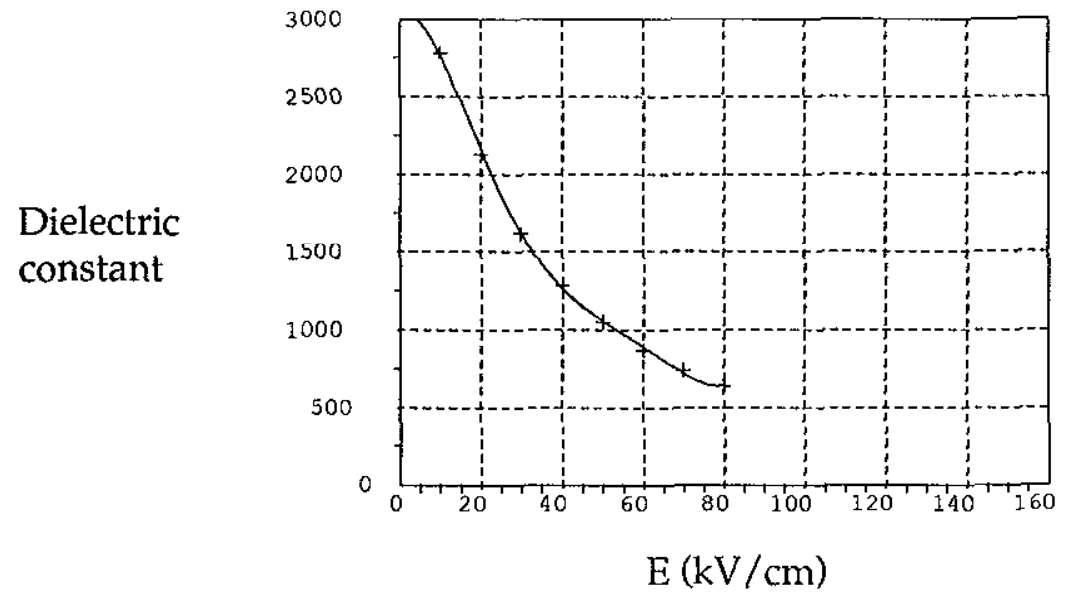

Where the curve is defined as:

$$
\begin{aligned}
& y=A+B x+C x^{2}+D x^{3}+E x^{4}+F x^{5} \\
& A=302902 \times 10^{3} \\
& B=130154 X 10^{1} \\
& C=-543349 \times 10^{0} \\
& D=1.67826 \times 10^{-1} \\
& E=-2.05422 \times 10^{-3} \\
& F=9.02243 \times 10^{-6}
\end{aligned}
$$




\subsection{Manufacture A material N4700 type corrected data}

Figure 4.3.1 The following figure illustrates the curve associated with manufacture $\mathbf{A}$ ceramic material similar type $\mathrm{N} 4700$ having a typical $k_{e}$ of 1250 . The functional relationship is $k_{e}$ as a function of average field stress (E) in $\mathrm{kV} / \mathrm{cm}$ of a selected specimen. The DUT failed at an average $\mathrm{E}$ of $66 \mathrm{kV} / \mathrm{cm}$. The vertical displacement is in dielectric constant and the horizontal displacement $\mathrm{E}$ is in $\mathrm{kV} / \mathrm{cm}$.

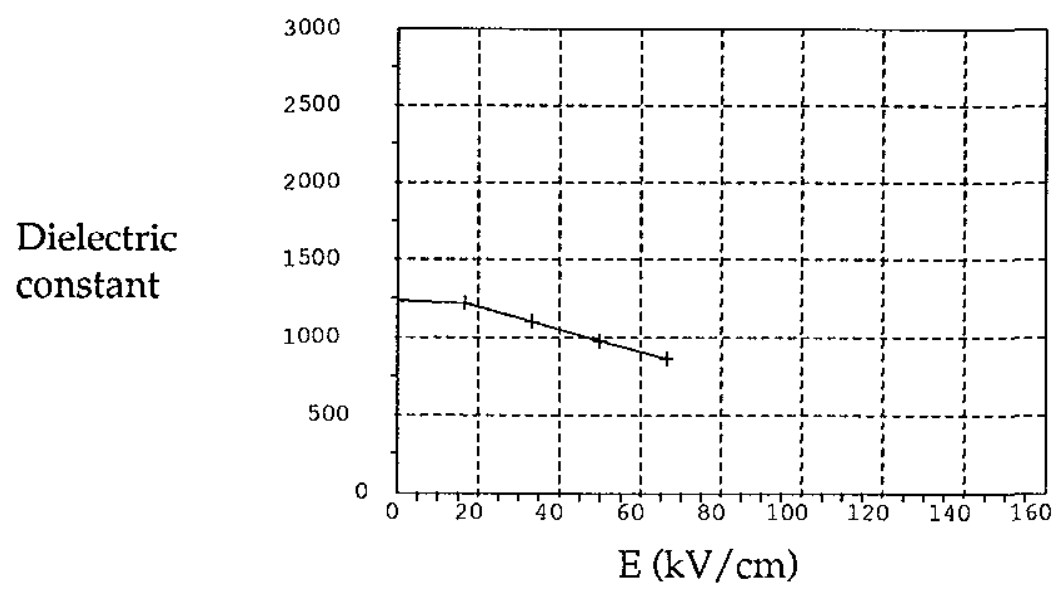

Figure 4.32 The following figure provides a third order curve fit to the above data and as a result more closely represents the anticipated values over the measured range.

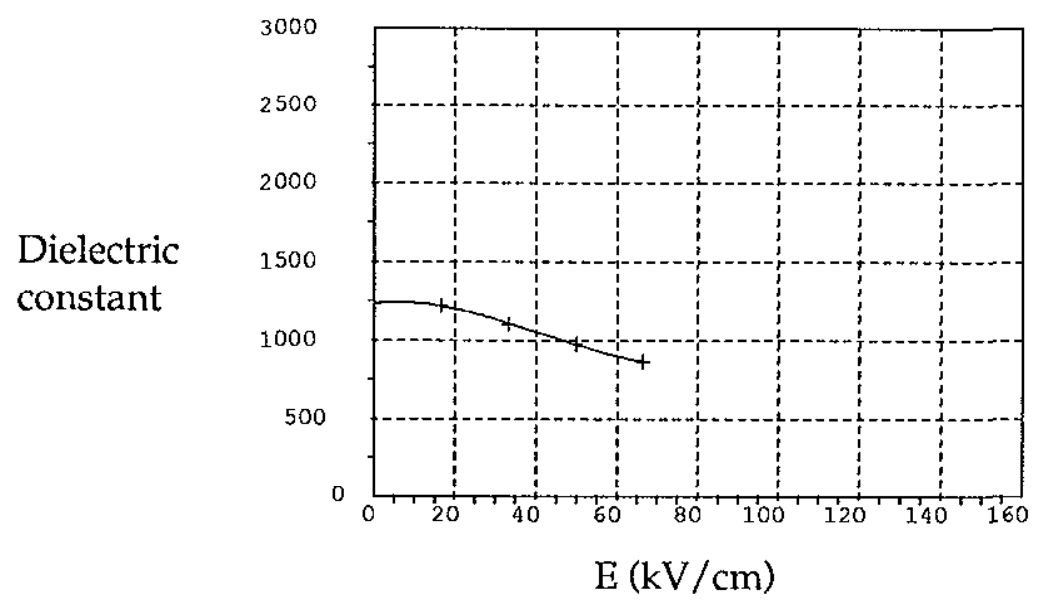

Where the curve is defined as:

$$
\begin{aligned}
& y=A+B x+C x^{2}+D x^{3} \\
& A=123523 \times 10^{3} \\
& B=2.64642 \times 10^{0} \\
& C=-263662 \times 10^{-1} \\
& D=2.10749 \times 10^{-3}
\end{aligned}
$$


4.4 Manufacture A material N2200 corrected data

Figure 441 The following figure illustrates the curve associated with manufacture $\mathbf{A}$ ceramic material type N2200 having a typical $k_{e}$ of 300 . The functional relationship is $k_{e}$ as a function of average field stress $(\mathrm{E})$ in $\mathrm{kV} / \mathrm{cm}$ of a selected specimen. The DUT failed at an average $\mathrm{E}$ of $400 \mathrm{kV} / \mathrm{cm}$. The vertical displacement is in dielectric constant and the horizontal displacement $\mathrm{E}$ is in $\mathrm{kV} / \mathrm{cm}$ This material remains flat until its intrinsic breakdown level.

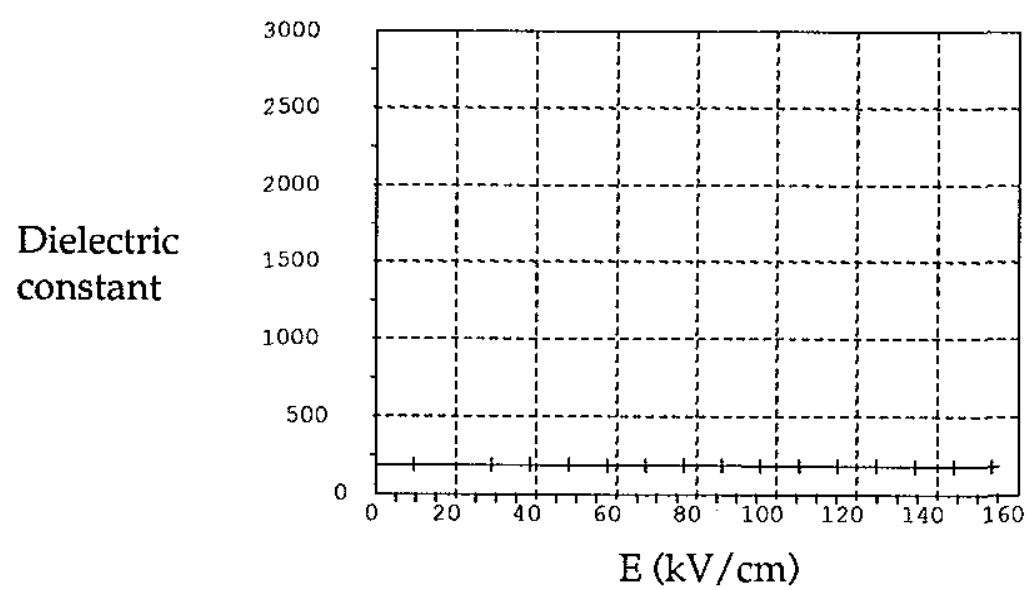

Figure 4.4.2 The following figure provides a fifth order curve fit to the above data and as a result more closely represents the anticipated values over the measured range

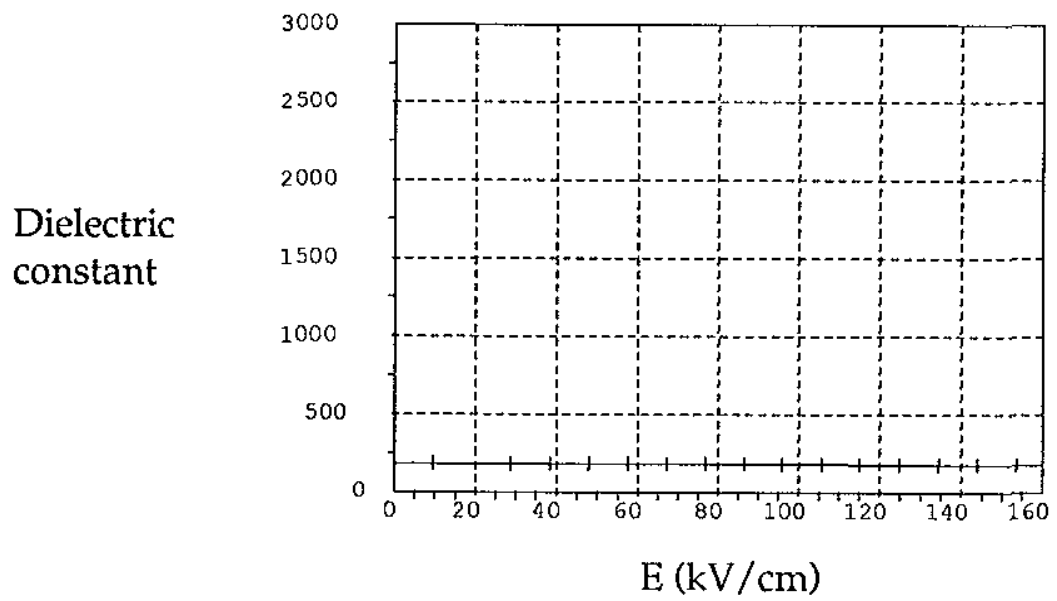

Where the curve is defined as:

$$
\begin{aligned}
& y=A+B x+C x^{2}+D x^{3}+E x^{4}+F x^{5} \\
& A=1.84001 X 10^{2} \\
& B=-2.01772 X 10^{-2} \\
& C=-4.61155 \times 10^{-5} \\
& D=-2.49486 \times 10^{-6} \\
& E=9.92680 X 10^{-9} \\
& F=-109073 \times 10^{-11}
\end{aligned}
$$




\subsection{Manufacture B material N4700 corrected data}

Figure 4.5.1 The following figure illustrates the curve associated with manufacture $B$ ceramic material type $\mathrm{N} 4700$ having a typical $k_{e}$ of 1650 The functional relationship is $k_{e}$ as a function of average field stress (E) in $\mathrm{kV} / \mathrm{cm}$ of a selected specimen. The DUT did not fail in this configuration because of the test specimen thickness and a limit of the bias HV supply of $100 \mathrm{kV}$. The vertical displacement is in dielectric constant and the horizontal displacement $\mathrm{E}$ is in $\mathrm{kV} / \mathrm{cm}$.

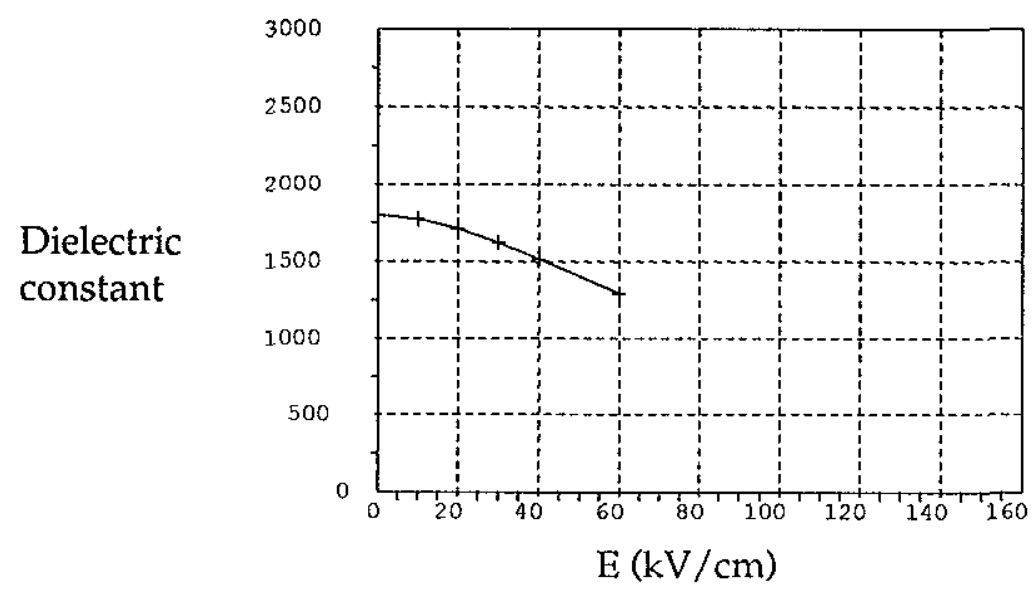

Figure 4.52 The following figure provides a fourth order curve fit to the above data and as a result more closely represents the anticipated values over the measured range.

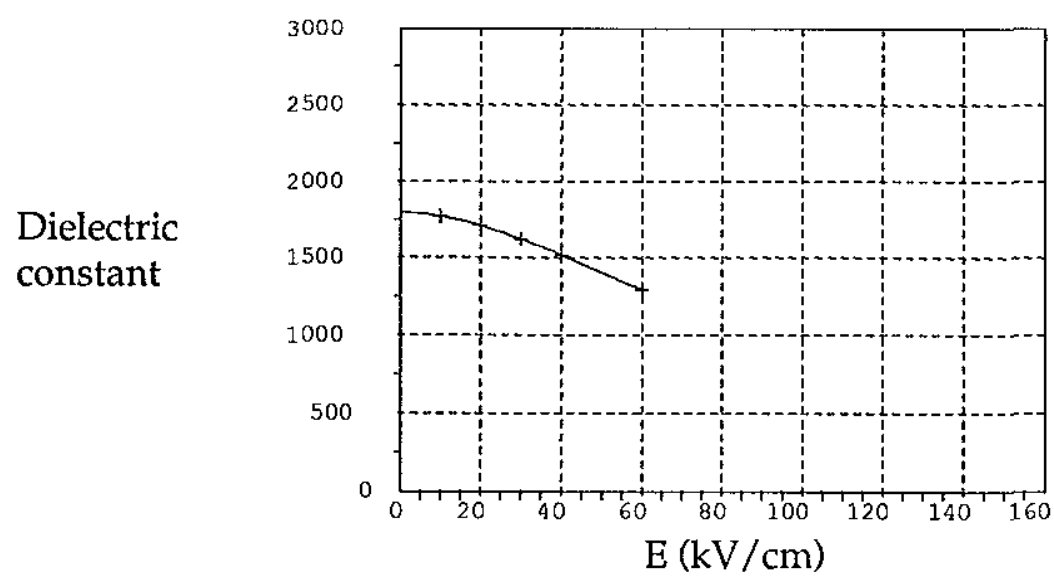

Where the curve is defined as:

$$
\begin{aligned}
& y=A+B x+C x^{2}+D x^{3}+E x^{4} \\
& A=179695 \times 10^{3} \\
& B=-6.06615 \times 10^{-1} \\
& C=-2.05900 \times 10^{-1} \\
& D=887579 \times 10^{-4} \\
& E=617138 \times 10^{-6}
\end{aligned}
$$


4.5.1 Manufacture B material N4700 corrected data

Figure 4.5.1.1The following figure illustrates the curve associated with manufacture $\mathbf{B}$ ceramic material type $\mathrm{N} 4700$ having a typical $k_{e}$ of 1850 . The functional relationship is $k_{e}$ as a function of average field stress (E) in $\mathrm{kV} / \mathrm{cm}$ of a selected specimen The DUT did fail in this configuration because the configuration was of an extended electrode contoured design. The vertical displacement is in dielectric constant and the horizontal displacement $\mathrm{E}$ is in $\mathrm{kV} / \mathrm{cm}$.

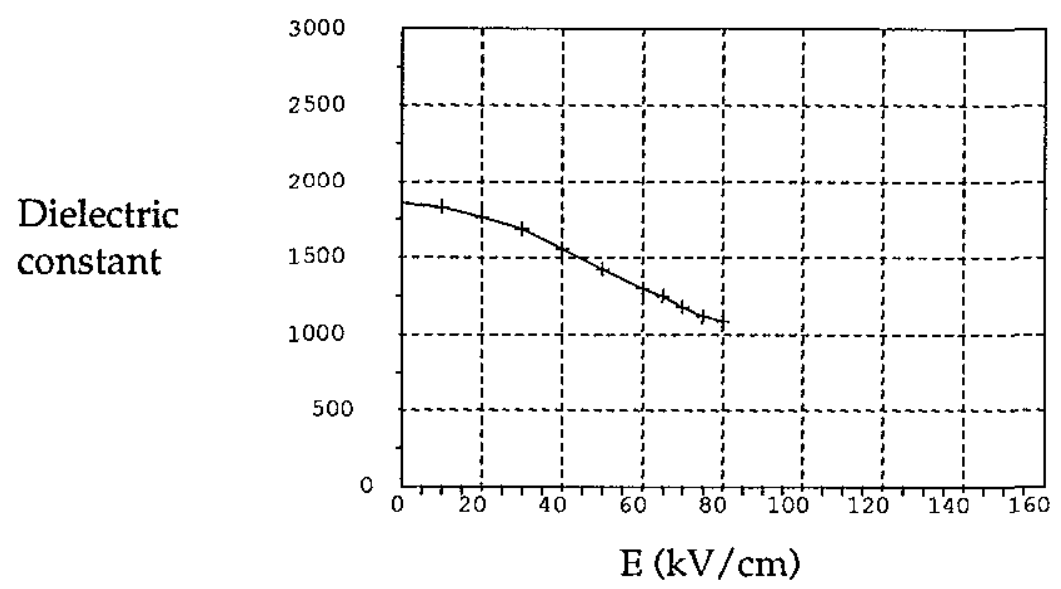

Figure 4.5.2.1The following figure provides a fourth order curve fit to the above data and as a result more closely represents the anticipated values over the entire range

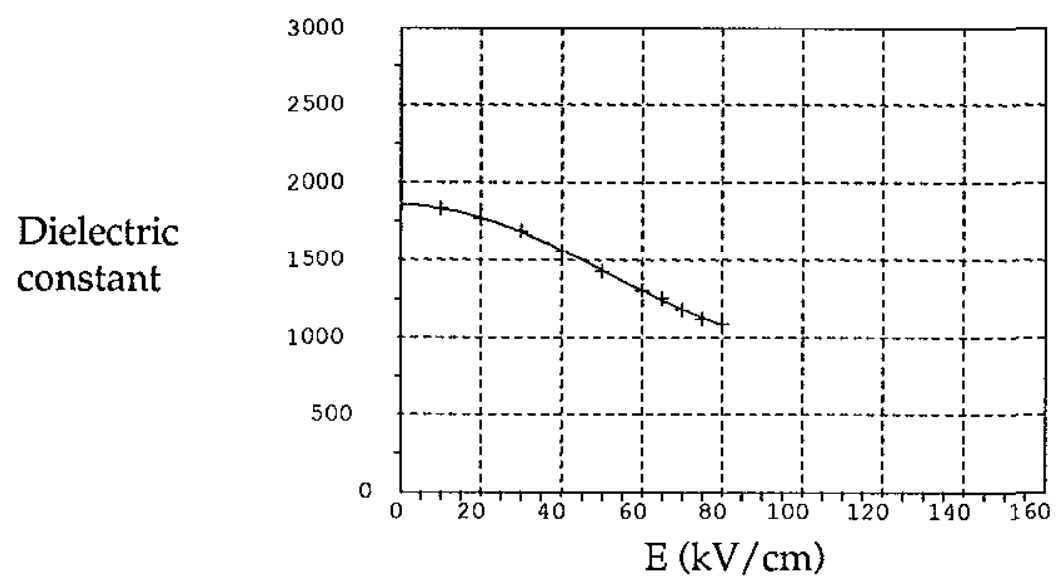

Where the curve is defined as.

$$
\begin{aligned}
& y=A+B x+C x^{2}+D x^{3}+E x^{4} \\
& A=185582 \times 10^{3} \\
& B=-906976 \times 10^{-1} \\
& C=-152559 \times 10^{-1} \\
& D=-147362 \times 10^{-3} \\
& E=3.81322 \times 10^{-5} \\
& F=-1.62360 \times 10^{-7}
\end{aligned}
$$


4.6 Manufacture B material N4700 corrected data

Figure 4.6.1 The following figure illustrates the curve associated with an alternative type of N4700 material supplied by manufacture B having a typical $k_{e}$ of 2600 The functional relationship is $k_{e}$ as a function of average field stress (E) in $\mathrm{kV} / \mathrm{cm}$ of a selected specimen. The DUT did not fail in this configuration because of the test specimen thickness and a limit of the bias HV supply of $100 \mathrm{kV}$ The vertical displacement is in dielectric constant and the horizontal displacement $\mathrm{E}$ is in $\mathrm{kV} / \mathrm{cm}$.

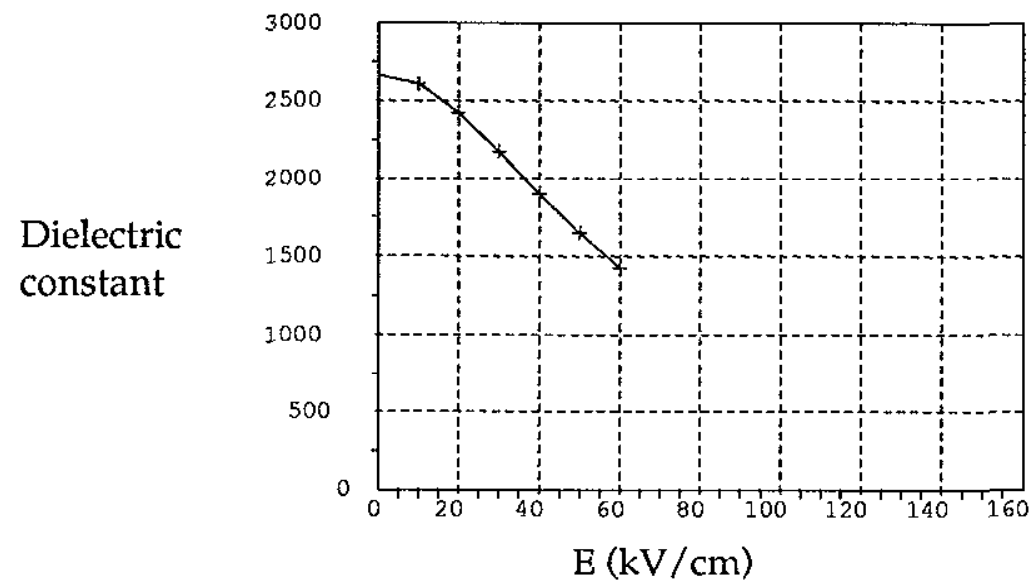

Figure 4.6.2 The following figure provides a fifth order curve fit to the above data and as a result more closely represents the anticipated values over the measured range.

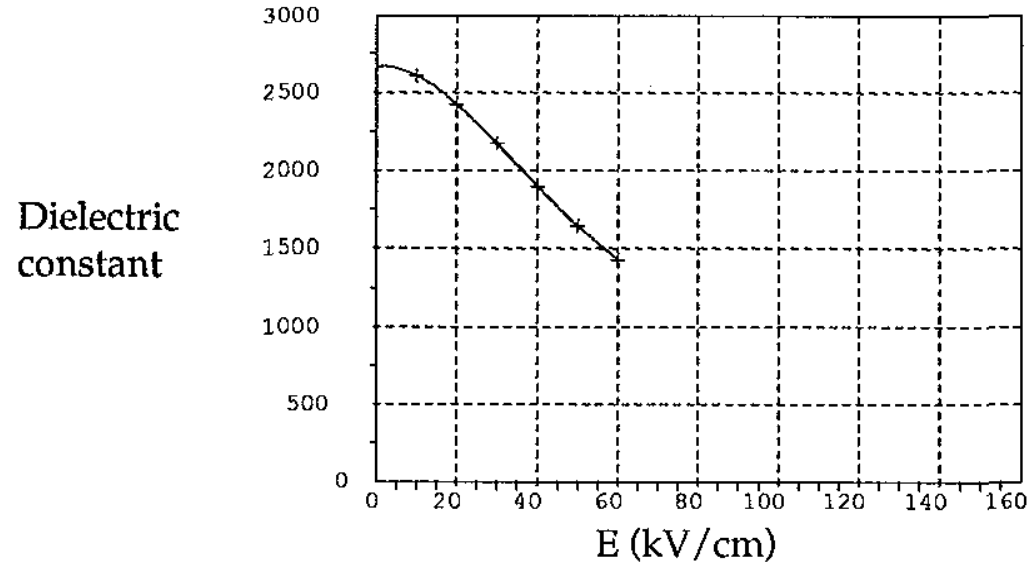

Where the curve is defined as:

$$
\begin{aligned}
& y=A+B x+C x^{2}+D x^{3}+E x^{4}+F x^{5} \\
& A=2.66416 \times 10^{3} \\
& B=305326 \times 10^{0} \\
& C=-9.78239 \times 10^{-1} \\
& D=1.11724 X 10^{-2} \\
& E=359809 \times 10^{-6} \\
& F=-4.58331 X 10^{-7}
\end{aligned}
$$




\section{Manufacture C material SR4700 corrected data}

Figure 4.7.1 The following figure illustrates the curve associated with manufacture $\mathbf{C}$ ceramic material type SR4700 having a typical $k_{e}$ of 2200 . The functional relationship is $k_{e}$ as a function of average field stress (E) in $\mathrm{kV} / \mathrm{cm}$ of a selected specimen The DUT failed at an average $E$ of $108 \mathrm{kV} / \mathrm{cm}$. The vertical displacement is in dielectric constant and the horizontal displacement $\mathrm{E}$ is in $\mathrm{kV} / \mathrm{cm}$

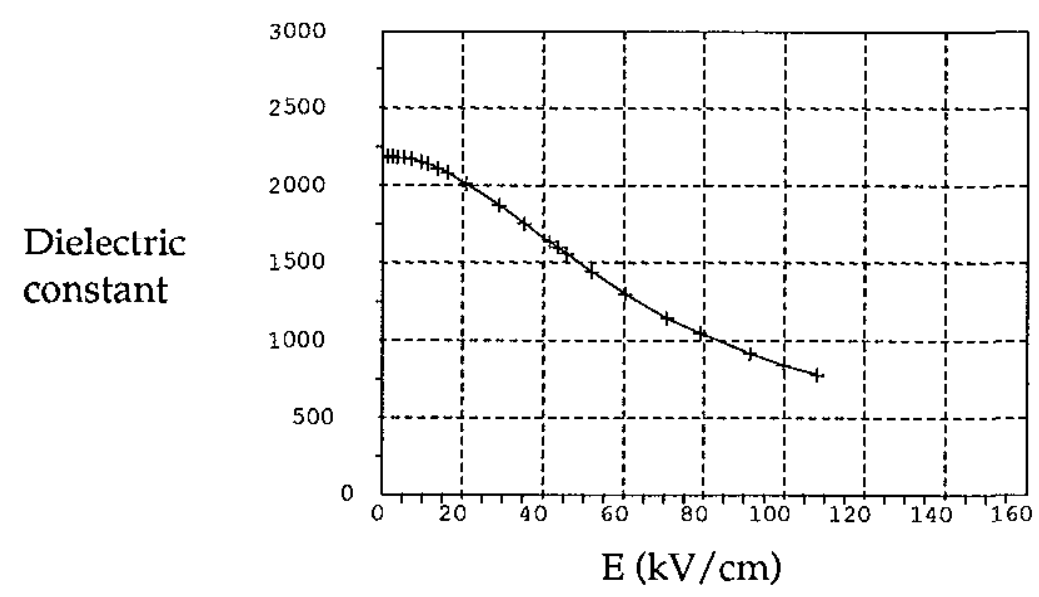

Figure 4.7.2 The following figure provides a fifth order curve fit to the above data and as a result more closely represents the anticipated values over the measured range.

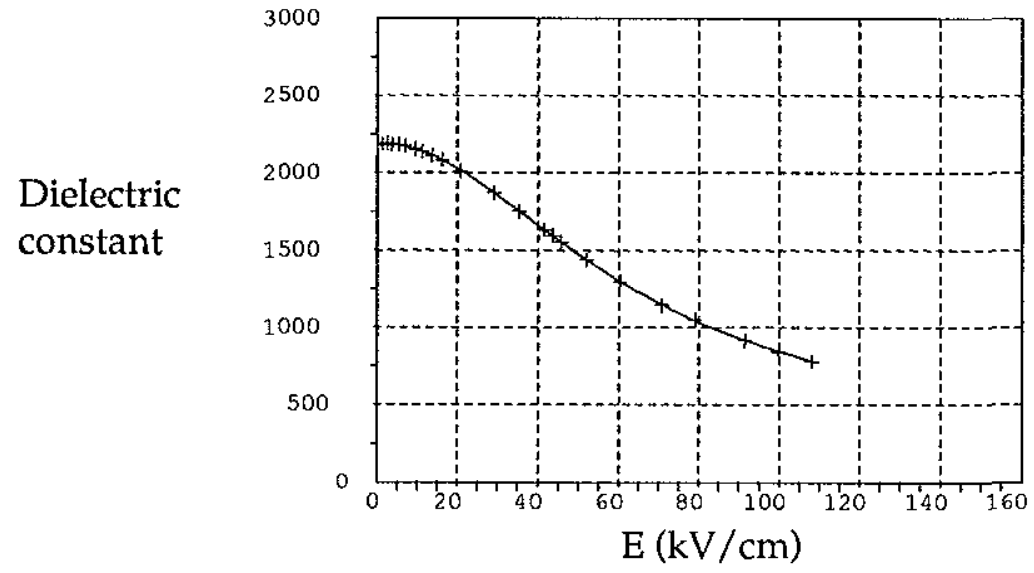

Where the curve is defined as:

$$
\begin{aligned}
& y=A+B x+C x^{2}+D x^{3}+E x^{4}+F x^{5} \\
& A=218043 \times 10^{3} \\
& B=2.91513 \times 10^{0} \\
& C=-7.07935 \times 10^{-1} \\
& D=9.74642 \times 10^{-3} \\
& E=-5.28992 \times 10^{-5} \\
& F=9.96148 \times 10^{-8}
\end{aligned}
$$




\subsection{Comparison of selected materials}

Figure 4.8.1 The following figure illustrates the curve associated with all the selected ceramic materials The functional relationship is $k_{e}$ as a function of average field stress (E) in $\mathrm{kV} / \mathrm{cm}$ of a selected specimen. The vertical displacement is in dielectric constant and the horizontal displacement $\mathrm{E}$ is in $\mathrm{kV} / \mathrm{cm}$.

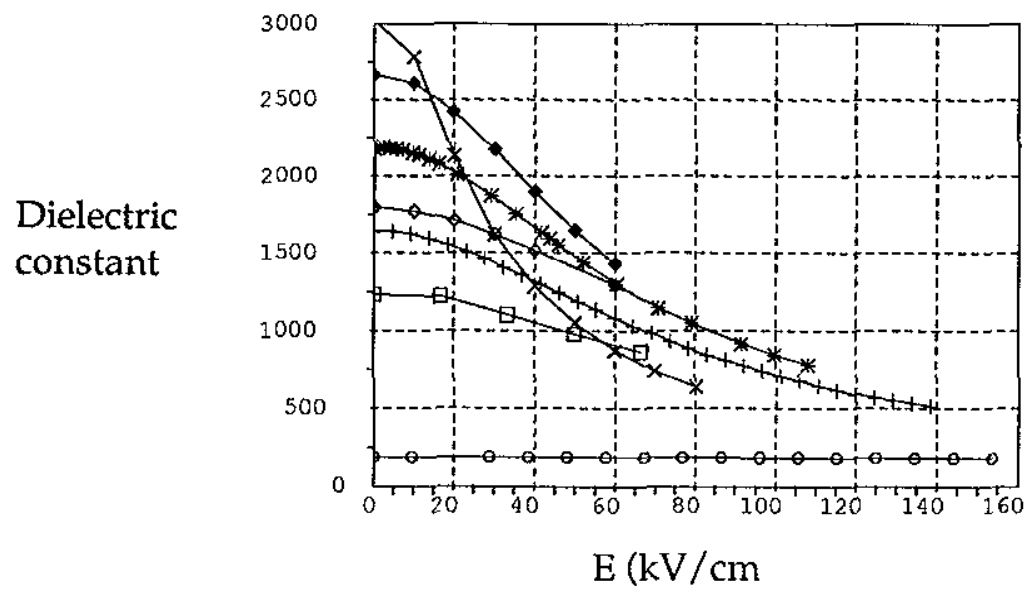

The above corresponding curve represents manutacture-estimated $k_{e}$ :

$\begin{array}{ll}1850 & + \\ 3000 & \mathrm{X} \\ 1250 & \square \\ 300 & \mathrm{O} \\ 1650 & 0 \\ 2600 & \bullet \\ 2200 & *\end{array}$




\subsection{Conclusion}

The effect of stressing high permittivity material causes a decrease in the resulting capacitance in the operating configuration. As a result the need to measure this parameter at the elevated stress levels is paramount to understanding circuit performance issues. A versatile process has been show to be easily performed on selected material and provided credible measurements at these elevated stress levels. Additional information of providing the functional information of the dielectric constant related to the average stress of the material under investigation adds to the hybrid, high-performance design scenarios. The selection of a gas environment to test the material removes problems previously shown to limit the bias level. This technique exceeds typically available biased capacitance measurement techniques and provides a critical engineering tool.

\subsection{References}

\footnotetext{
${ }^{1}$ Michael J Wilson, David A Goerz, and Ron D Speer, LCR Bridge Measurements at Elevated Operating Levels, presented at the 1997 High-Voltage Workshop, May 5-8, 1997, Newport Beach, CA, Lawrence Livermore National Laboratory, Livermore, CA, UCRL-MI-125796, (1997).

${ }^{2}$ William J Sarjeant, Energy Storage Capacitors, Los Alamos National Laboratory, NM, LA-UR-79-1044, (1979)

${ }^{3}$ Michael J Wilson and David A Goerz, "Compact High-Voltage Structures," in $11^{\text {th }}$ IEEE International Pulsed Power Conference, Baltimore, MD, June 29 - July 2, 1997, or Lawrence Livermore National Laboratory, Livermore, CA, UCRL-JC-125862 (1997)

${ }^{4}$ David A Goerz, Michael J Wilson, and Ronnie D Speer, "A Low-Profile High-Voltage Compact Gas Switch, "in $11^{\text {th }}$ IEEE International Pulsed Power Conference, Baltimore, MD, June 29 - July 2, 1997, or Lawrence Livermore National Laboratory, Livermore, CA UCRL-JC-127763, (1997).
} 


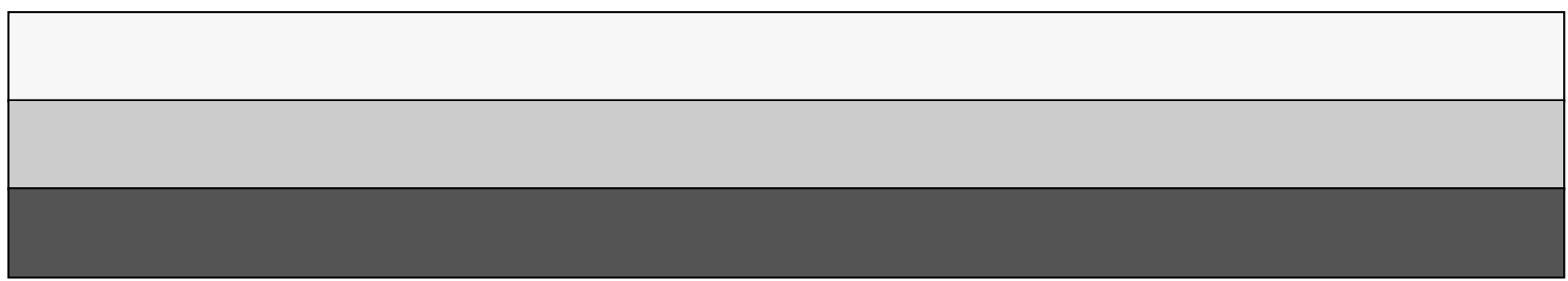

